\title{
Aquaporin 3 protects against lumbar intervertebral disc degeneration via the $\mathrm{Wnt} / \boldsymbol{\beta}$-catenin pathway
}

\author{
HUANXIN XIE, YONGBIN JING, JINGJUN XIA, XINTAO WANG, CHANGCHENG YOU and JINGLONG YAN \\ Department of Orthopedic Surgery, The Second Affiliated Hospital \\ of Harbin Medical University, Harbin, Heilongjiang 150001, P.R. China
}

Received April 8, 2015; Accepted January 5, 2016

DOI: $10.3892 /$ ijmm.2016.2470

\begin{abstract}
Previous studies have demonstrated that the expression of aquaporin 3 (AQP3), a water channel which promotes glycerol permeability and water transport across cell membranes, is reduced in degenerative lumbar intervertebral disc (IVD) tissues. However, the role of AQP3 in the pathogenesis of IVD degeneration has not recieved much scholarly attention. The objective of the present study was to investigate the effect of AQP3 on cell proliferation and extracellular matrix (ECM) degradation in human nucleus pulposus cells (hNPCs) using gain-of-function and loss-offunction experiments, and to determine whether Wnt/ $\beta$-catenin signaling is involved in the effect of AQP3 on IVD degeneration. hNPCs were transfected with the AQP3-pcDNA3.1 plasmid or AQP3 siRNA to overexpress or suppress AQP3. An MTT assay was performed to determine cell proliferation, and we found that AQP3 promoted hNPC proliferation. The expression of aggrecan, a disintegrin and metalloproteinase with thrombospondin motifs (ADAMTS) 4 and ADAMTS5 was detected using western blot analysis, to examine the effect of AQP3 on ECM degradation in hNPCs. The results revealed that AQP3 inhibited ECM degradation in hNPCs. In addition, we found that $\mathrm{Wnt} / \beta$-catenin signaling was suppressed by AQP3. However, the effect of AQP3 on hNPC proliferation and ECM degradation was reversed by treatment with lithium chloride, a known activator of Wnt/ $\beta$-catenin signaling. In conclusion, using in vitro and in vivo tests, we have reported for the first time, to the best of our knowledge, that AQP3 exerts protective effects against IVD degeneration, and these are effected, at least partially, through the inhibition of Wnt/ $\beta$-catenin signaling.
\end{abstract}

Correspondence to: Professor Jinglong Yan, Department of Orthopedic Surgery, The Second Affiliated Hospital of Harbin Medical University, 246 Xuefu Street, Nangang, Harbin, Heilongjiang 150001, P.R. China

E-mail: jinglong_y@163.com

Key words: aquaporin 3, wnt/ $\beta$-catenin, intervertebral disc degeneration, proliferation, extracellular matrix

\section{Introduction}

Lumbar intervertebral disc (IVD) degeneration is a major cause of lower back pain (1); it may reduce the quality of life of individuals (2) and causes significant socioeconomic problems (3). During the process of degeneration, IVDs undergo morphological and biochemical changes $(4,5)$. A decrease in water content is one of the characteristics of lumbar IVD degeneration (6).

Aquaporins (AQPs) are a family of small, integral membrane proteins which regulate the influx and outflow of water and small molecules (7). AQP3 is a member of the AQP family, and it acts as a water channel to promote glycerol permeability and water transport across cell membranes (8). It has previously been noted that AQP3 immunoreactivity significantly decreased in the nucleus pulposus (NP) and annulus fibrosus (AF) of aged rats compared with young rats (9), and the expression of AQP3 is reduced in the degenerated tissue of human lumbar IVDs (10). However, little is known about the effect of AQP3 on the pathogenesis of IVD degeneration.

Wnt/ $\beta$-catenin signaling plays an important role in the progression of IVD degeneration (11-13). The activation of Wnt/ $\beta$-catenin signaling triggers the process of IVD degeneration, suppresses the proliferation of NP cells and induces cellular senescence (14-16).

In the present study, we performed an in vitro study using human NP cells (hNPCs) to evaluate the effect of AQP3 on the progression of IVD degeneration for the first time, to the best of our knowledge. Furthermore, this experiment aimed to determine whether Wnt $/ \beta$-catenin signaling is involved in the effect of AQP3 on IVD degeneration

\section{Materials and methods}

Tissue samples. The present study was approved by the Ethics Committee of The Second Affiliated Hospital of Harbin Medical University (Harbin, China). Following the provision of informed consent, patients with lumbar IVD degeneration $(n=12)$ and patients with spinal trauma $(n=8)$ who underwent surgery at the Department of Orthopedic Surgery, The Second Affiliated Hospital of Harbin Medical University were enrolled in this study. The human NP samples were harvested and immediately frozen in liquid nitrogen for RNA and protein extraction at a later date. 
Cell culture. hNPCs (ScienCell Research Laboratories, San Diego, CA, USA) were maintained in Dulbecco's modified Eagle's medium (DMEM) supplemented with $10 \%$ fetal bovine serum (FBS) (both from Gibco Life Technologies, Carlsbad, $\mathrm{CA}, \mathrm{USA})$ at $37^{\circ} \mathrm{C}$ in a humidified atmosphere of $5 \% \mathrm{CO}_{2}$. Lithium chloride $(\mathrm{LiCl})$ was purchased from Sigma-Aldrich (St. Louis, MO, USA) and dissolved in water at a concentration of $20 \mathrm{mM}$, to treat hNPCs for $24 \mathrm{~h}$.

Cell transfection. The cells were seeded at $2.5 \times 10^{5}$ cells/well in a 6-well plate. Following overnight adherence, the cells were transfected with $1 \mu \mathrm{g}$ AQP3-pcDNA3.1 plasmid, empty pcDNA3.1 vector, AQP3 siRNA or control siRNA (all were obtained from Fitgene Biological Technology Co., Ltd., Guangzhou, China) per well using Lipofectamine 2000 (Invitrogen Life Technologies, Carlsbad, CA, USA) according to the manufacturer's instructions. Cells in the blank group were not transfected with any plasmids or siRNA. Briefly, plasmid DNA/siRNA and Lipofectamine 2000 (Invitrogen Life Technologies) were diluted in two independent $250 \mu \mathrm{l}$ volumes of Opti-MEM I (Invitrogen Life Technologies). After a 5-min incubation at room temperature, the DNA and the Lipofectamine 2000 in Opti-MEM I were combined and incubated for an additional 20 min to allow for the formation of DNA-Lipofectamine 2000 complexes. The DNA-Lipofectamine 2000 complexes were then added to each well. Six hours post-transfection, the medium was replaced with $2 \mathrm{ml}$ of complete growth medium.

Reverse transcription-quantitative polymerase chain reaction (RT-qPCR). Total RNA was extracted from the tissues using TRIzol (Invitrogen Life Technologies). Subsequently, $1 \mu \mathrm{g}$ of total RNA was reverse transcribed to cDNA using M-MLV reverse transcriptase (Promega Corp., Madison, WI, USA). The qPCR reactions were performed using the SYBR-Green PCR Master Mix (Applied Biosystems Life Technologies; Thermo Fisher Scientific, Waltham, MA, USA). The PCR primers were as follows: AQP3 sense, 5'-ccttcttgggtgctggaata-3' and antisense, 5'-acacacacgataagggaggc-3'; and $\beta$-actin sense, 5'-aagtactccgtgtggatcgg-3' and antisense, 5'-caccttcaccgttccagttt-3'. The PCR cycles were as follows: initial denaturation at $95^{\circ} \mathrm{C}$ for $5 \mathrm{~min}$, followed by 40 cycles of $94^{\circ} \mathrm{C}$ for $20 \mathrm{sec}, 60^{\circ} \mathrm{C}$ for $20 \mathrm{sec}$ and $72^{\circ} \mathrm{C}$ for $5 \mathrm{~min}$, and a final extension at $72^{\circ} \mathrm{C}$ for $5 \mathrm{~min}$. The PCR products were confirmed by electrophoresis on $2 \%$ agarose gel. All reactions were performed in duplicate on an ABI PRISM 7900HT Sequence Detection system (Applied Biosystems Life Technologies). The expression of mRNA was defined from the threshold cycle, and the final results were expressed as foldchanges between the patients with lumbar IVD degeneration and the patients with spinal trauma after normalization to the internal control $\beta$-actin.

3-(4,5-Dimethylthiazol-2-yl)-2,5-diphenyltetrazolium bromide (MTT) assay. In the present study, an MTT assay was carried out using a cell proliferation assay kit (Boster Inc., Wuhan, China). Briefly, $10^{4}$ cells were plated on 96-well plates. Following treatment, $20 \mu \mathrm{l}$ MTT solution was added to each well and incubated for $4 \mathrm{~h}$ at $37^{\circ} \mathrm{C}$, followed by the addition of $100 \mu \mathrm{l}$ dimethyl sulfoxide (DMSO; Sigma-Aldrich) to solubilize the formazan. Finally, absorbance was recorded at $570 \mathrm{~nm}$ using a Multiskan Ascent 354 microplate reader (Thermo
Labsystems, Waltham, MA, USA). The cell viability results of three independent experiments were normalized to the cells treated with medium only.

Western blot analysis. Proteins were prepared using a total protein extraction kit (KeyGen Biotech, Nanjing, China) according to the manufacturer's instructions. The proteins were resolved on a sodium dodecyl sulfate (SDS) polyacrylamide gel and electrotransferred to a nitrocellulose membrane (EMD Millipore, Billerica, MA, USA). After blocking with 5\% non-fat milk in TBST at $4^{\circ} \mathrm{C}$ overnight, the membranes were incubated with the following specific antibodies: rabbit polyclonal to aquaporin 3 (ab125219; 1:400); rabbit polyclonal to aggrecan (ab36861; 1:500); rabbit polyclonal to a disintegrin and metalloproteinase with thrombospondin motifs (ADAMTS) 4 (ab45038; 1:1,000); rabbit polyclonal to ADAMTS5 (ab41037; 1:400); rabbit polyclonal to Axin 2 (ab32197; 1:500) (all from Abcam, Cambridge, MA, USA); rabbit polyclonal to $\beta$-catenin (sc-7199; 1:800; Santa Cruz Biotechnology, Inc., Santa Cruz, CA, USA); rabbit polyclonal to $\beta$-actin (RS-0061R; 1:1,000; Shanghai Ruiqi Biological Technology Co., Ltd., Shanghai, China) followed with goat anti-rabbit IgG polyclonal antibody (020002-G; 1:5,000; Beijing CellChip Biotechnology, Beijing, China). Immunolabeling was undertaken using an ECL western blotting kit (Pierce Biotechnology, Inc., Rockford, IL, USA).

Statistical analysis. Data are presented as the means \pm standard deviation (SD) from at least three independent experiments conducted in triplicate. The differences between two groups were analyzed by Student's t-test. P-values $<0.05$ were considered to indicate a statistically significant difference.

\section{Results}

AQP3 expression in the NP tissue samples. AQP3 expression in the NP tissues was examined at the mRNA and protein levels. RT-qPCR results revealed that AQP3 mRNA expression was significantly decreased in the samples from patients with IVD degenereration compared with that in the control samples (the patients with spinal trauma). Similar to the mRNA expression results, AQP3 protein expression was also lower in the NP tissues from patients with IVD degeneration than those of patients in the control group (the patients with spinal trauma) (Fig. 1).

Expression of AQP3 in human NP cells following transfection with the AQP3-pcDNA3.1 plasmid and AQP3 siRNA. AQP3pcDNA3.1 plasmid or AQP3-siRNA were transfected into hNPCs to cause overexpression or suppression of AQP3. Empty pcDNA3.1 vector and control siRNA exerted no significant effects on AQP3 expression (Fig. 2). However, AQP3 protein levels in the hNPCs were significantly increased by transfection with the AQP3-pcDNA3.1 plasmid. Transfection with the AQP3 siRNA resulted in a significant decrease in AQP3 expression.

Effect of AQP3 expression on hNPC proliferation. An MTT assay was performed to determine the effect of AQP3 overexpression on hNPC proliferation. AQP3 overexpression significantly increased hNPC proliferation (Fig. 3). In order to confirm the effect of endogenous AQP3 on hNPC proliferation, we examined whether AQP3 knockdown by AQP3 siRNA 

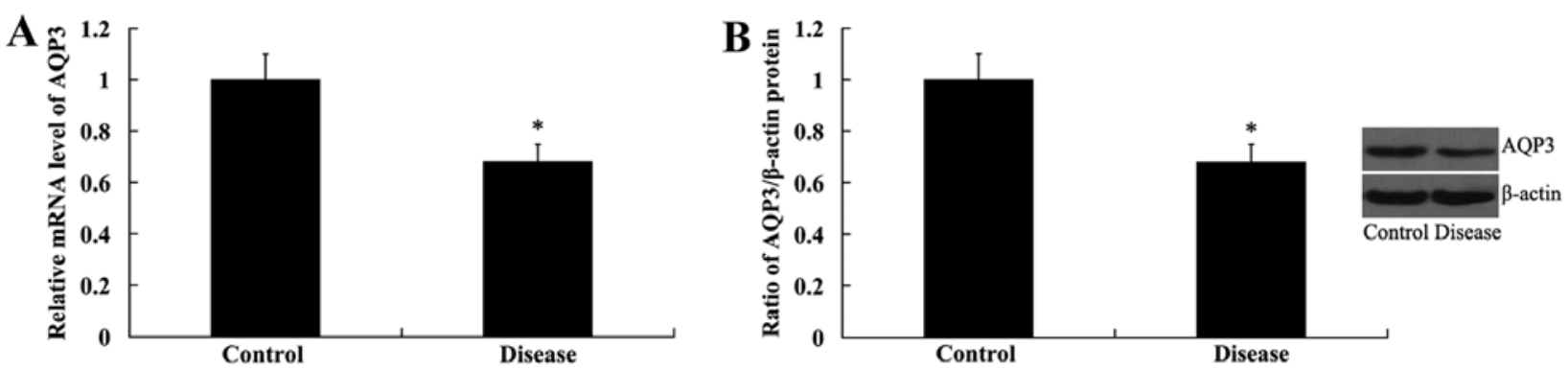

Figure 1. Expression of aquaporin 3 (AQP3) in the nucleus pulposus tissues of patients with lumbar intervertebral disc (IVD) degeneration. (A) Relative mRNA level. (B) Relative protein level. " $\mathrm{P}<0.05$. The patients with lumbar IVD degeneration were in the disease group, and the patients with spinal trauma were in the control group.
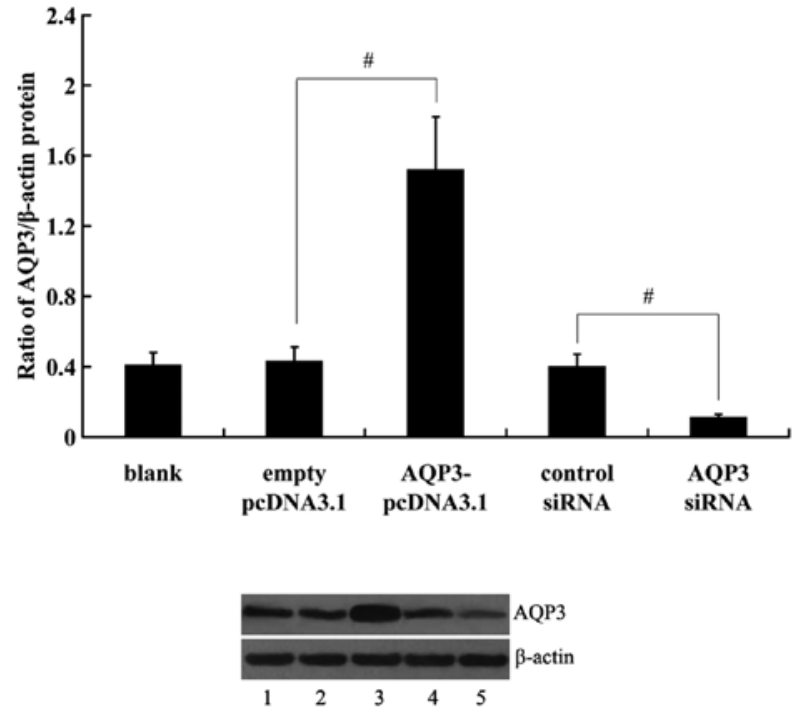

Figure 2. Expression of aquaporin 3 (AQP3) in human nucleus pulposus cells (hNPCs) following transfection with the AQP3-pcDNA3.1 plasmid and AQP3 siRNA. "P<0.01. Lane 1, blank group; lane 2, empty pcDNA3.1 group; lane 3 , AQP3-pcDNA3.1 group; lane 4, control siRNA group; lane 5, AQP3 siRNA group. Cells in the blank group were not transfected with any plasmids or SiRNA.

affected the survival rate of hNPCs. It was demonstrated that AQP3 knockdown exerted a significant inhibitory effect on hNPC proliferation.

Effect of AQP3 expression on extracellular matrix (ECM) degradation. The effect of AQP3 overexpression on ECM degradation was investigated by western blot analysis. The results demonstrated that aggrecan expression was increased in AQP3 overexpressing-hNPCs (Fig. 4A and B). In addition, the expression of the enzymes which are involved in the matrix degrading process, ADAMTS4 and ADAMTS5, was significantly decreased in hNPCs following overexpression of AQP3 (Fig. 4A, C and D).

The effect of endogenous AQP3 on ECM degradation was also determined by detecting the expression of aggrecan, ADAMTS4 and ADAMTS5 in hNPCs. We demonstrated that the expression level of aggrecan was significantly decreased in hNPCs following transfection with the AQP3 siRNA (Fig. 4A and B). Moreover, knockdown of AQP3 expression in hNPCs led to the increased expression of ADAMTS4 and ADAMTS5 (Fig. 4A, C and D).

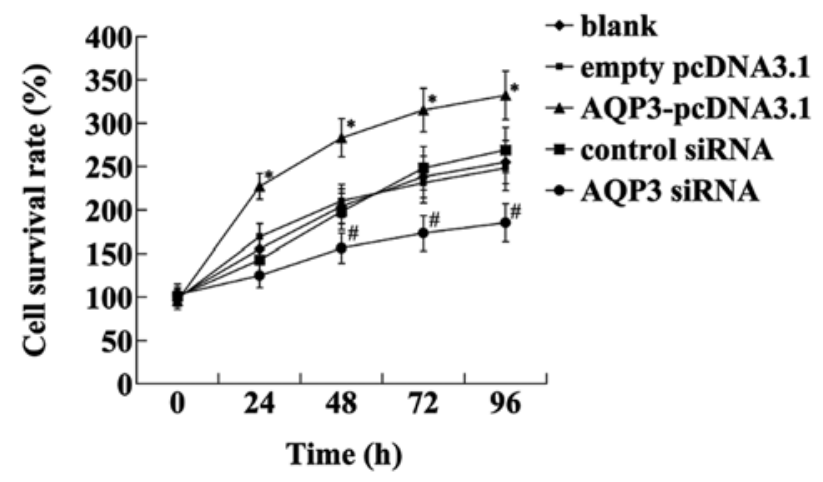

Figure 3. Effect of aquaporin 3 (AQP3) expression on human nucleus pulposus cell (hNPC) proliferation. ${ }^{*} \mathrm{P}<0.05$ compared with empty pcDNA3.1; $\# \mathrm{P}<0.05$ compared with control siRNA.

Effect of AQP3 on Wnt/ $\beta$-catenin signaling. To investigate the effect of AQP3 overexpression on $\mathrm{Wnt} / \beta$-catenin signaling, $\beta$-catenin and Axin 2 protein expression was measured in hNPCs. The results from western blot analysis revealed that AQP3 overexpression significantly decreased the expression of $\beta$-catenin and Axin 2. Knockdown of AQP3 expression in hNPCs induced a significant increase in $\beta$-catenin and Axin 2 protein levels (Fig. 5).

Wnt/ $\beta$-catenin signaling activation reverses the effect of $A Q P 3$ on $h N P C$ proliferation and ECM degradation. To investigate whether Wnt/ $\beta$-catenin signaling was involved in mediating the effect of AQP3 on hNPC proliferation and ECM degradation, hNPCs overexpressing AQP3 were treated with $\mathrm{LiCl}$, a known activator of Wnt signaling. As shown in Fig. 6, in hNPCs the protein levels of $\beta$-catenin and Axin 2, which had been downregulated by AQP3 overexpression, were significantly increased by incubation with $20 \mathrm{mM} \mathrm{LiCl}$ for $24 \mathrm{~h}$. An MTT assay revealed that the effect of AQP3 overexpression on promoting hNPC proliferation was attenuated by $\mathrm{LiCl}$ (Fig. 7). In addition, we noted that the altered expression of aggrecan, ADAMTS4 and ADAMTS5 in AQP3-overexpressing hNPCs was reversed by $\mathrm{LiCl}$ (Fig. 8).

\section{Discussion}

AQP3 is expressed in multiple epithelial tissues, knee articular cartilage, subchondral osteoblasts, synovium and IVDs $(17,18)$. In the present study, we found that AQP3 was downregulated 

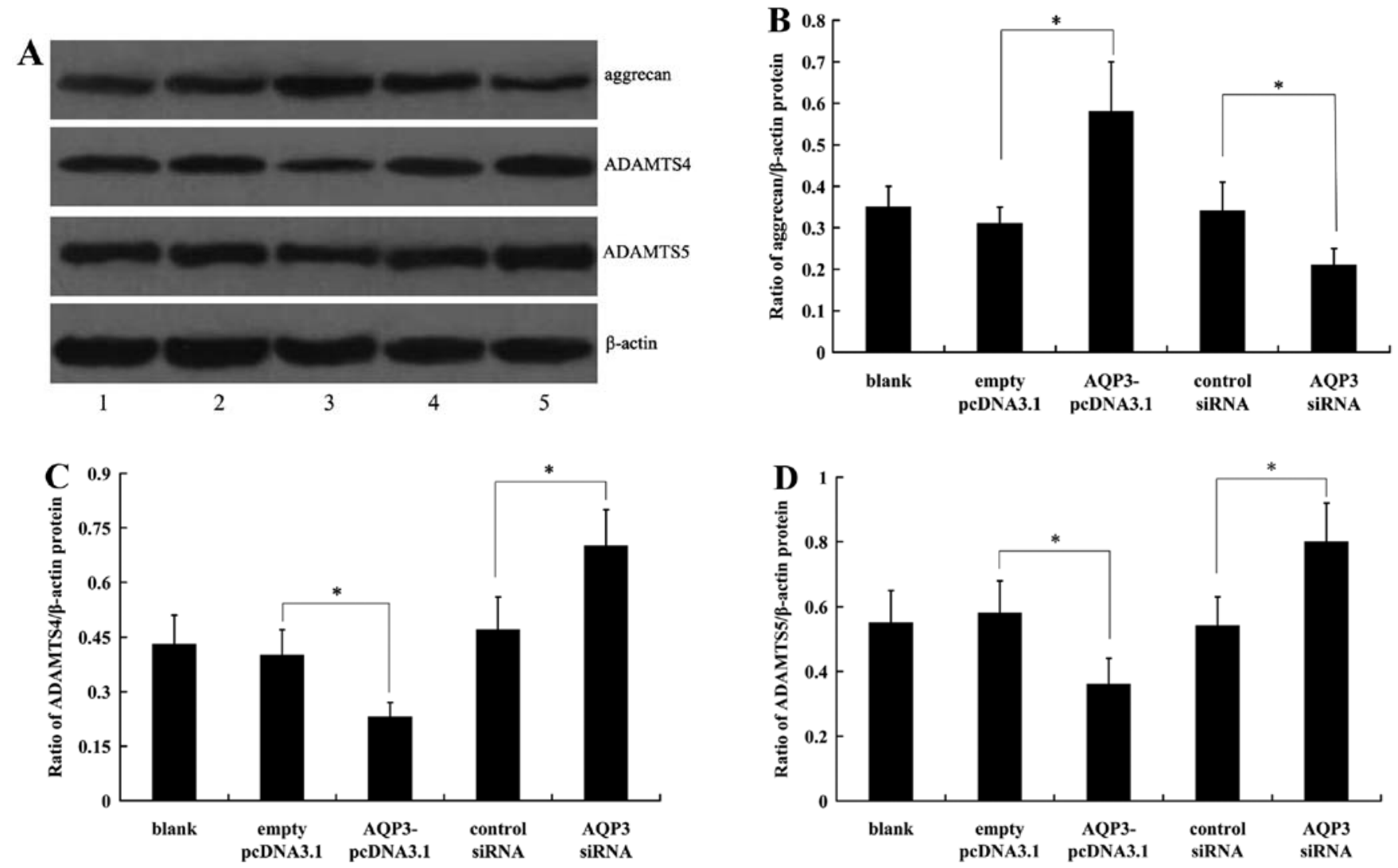

Figure 4. Effect of aquaporin 3 (AQP3) expression on extracellular matrix (ECM) degradation. (A) Western blot analysis of aggrecan, a disintegrin and metalloproteinase with thrombospondin motifs (ADAMTS) 4 and ADAMTS5. Relative protein levels of (B) aggrecan, (C) ADAMTS4 and (D) ADAMTS5. "P<0.05. Lane 1, blank group; lane 2, empty pcDNA3.1 group; lane 3, AQP3-pcDNA3.1 group; lane 4, control siRNA group; lane 5, AQP3 siRNA group. Cells in the blank group were not transfected with any plasmids or siRNA.

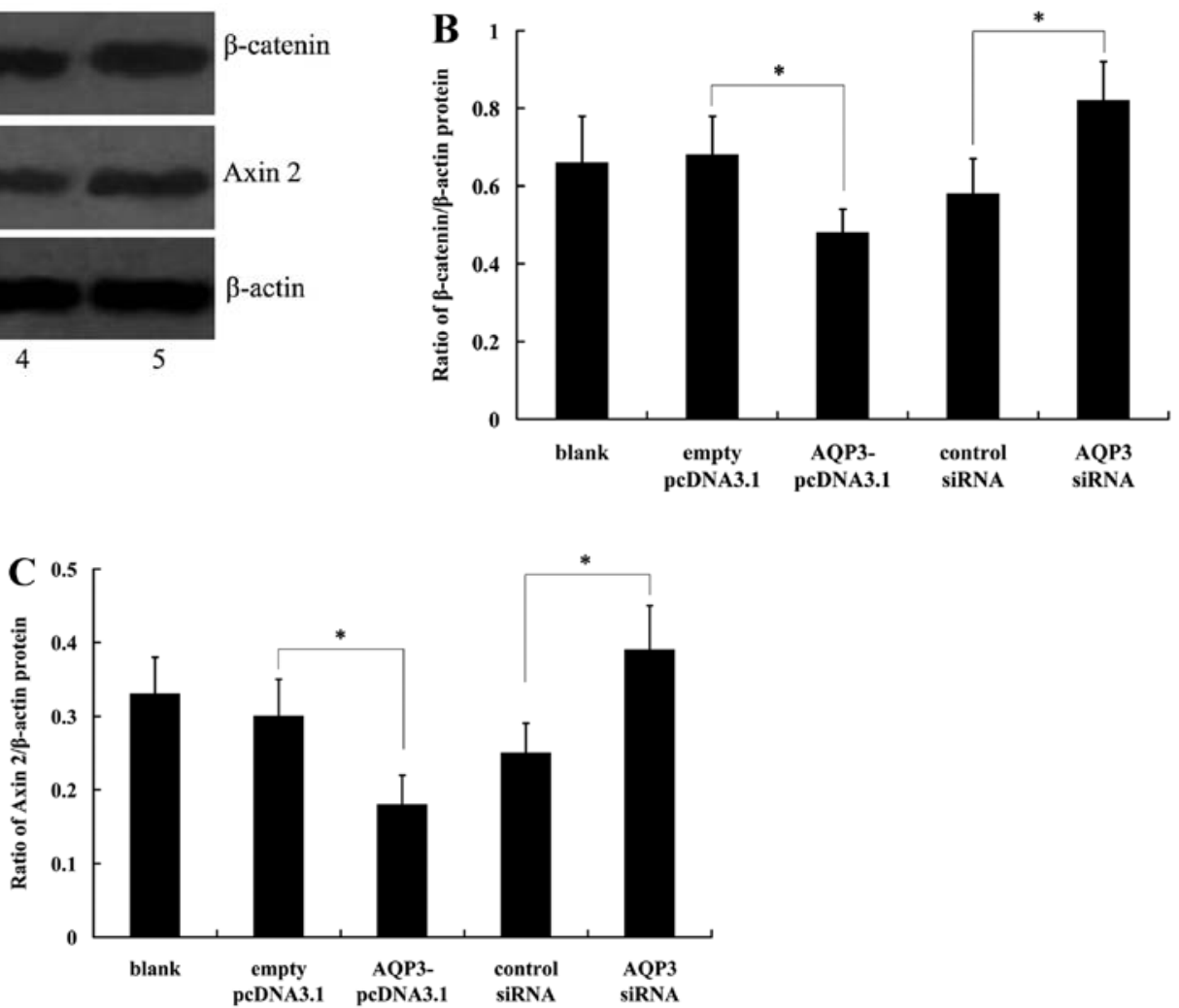

Figure 5. Effect of aquaporin 3 (AQP3) expression on Wnt/ $\beta$-catenin signaling. (A) Western blot analysis of $\beta$-catenin and Axin 2 . Relative protein levels of (B) $\beta$-catenin and (C) Axin 2. "P<0.05. Lane 1, blank group; lane 2, empty pcDNA3.1 group; lane 3, AQP3-pcDNA3.1 group; lane 4, control siRNA group; lane $5, \mathrm{AQP} 3$ siRNA group. Cells in the blank group were not transfected with any plasmids or siRNA. 


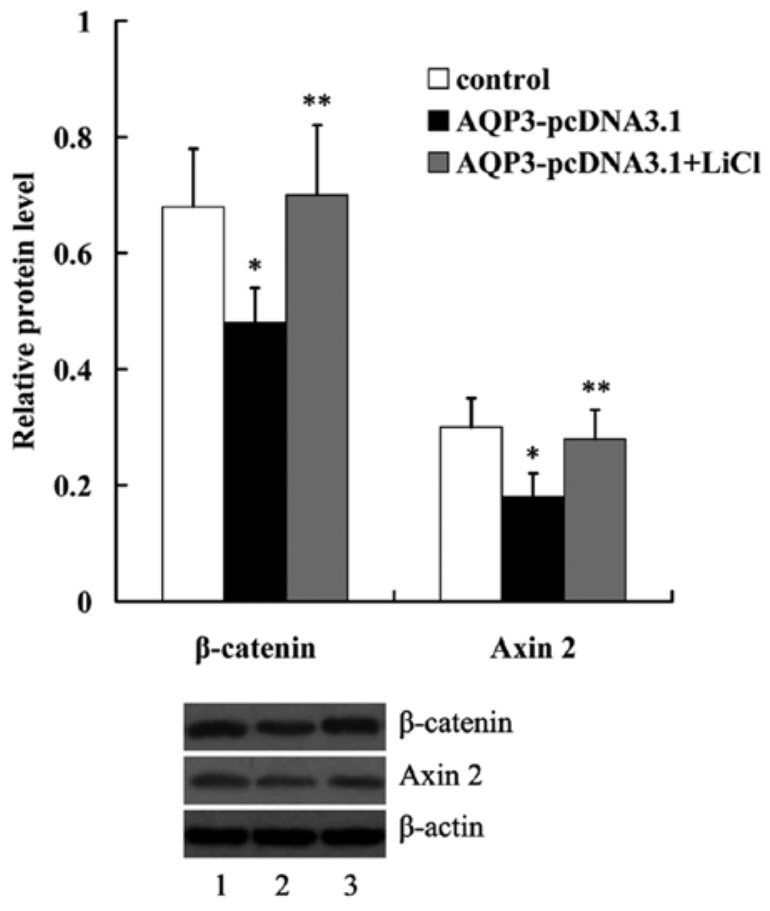

Figure 6. Expression of $\beta$-catenin and Axin 2 in human nucleus pulposus cells (hNPCs) following transfection with the AQP3-pcDNA3.1 plasmid and treatment with lithium chloride $(\mathrm{LiCl}) .{ }^{*} \mathrm{P}<0.05$ compared with the control group; ${ }^{* *} \mathrm{P}<0.05$ compared with the AQP3-pcDNA3.1 group. Lane 1, control group; lane 2, AQP3-pcDNA3.1 group; lane 3, AQP3-pcDNA3.1 + LiCl group. $\mathrm{AQP} 3$, aquaporin 3 .

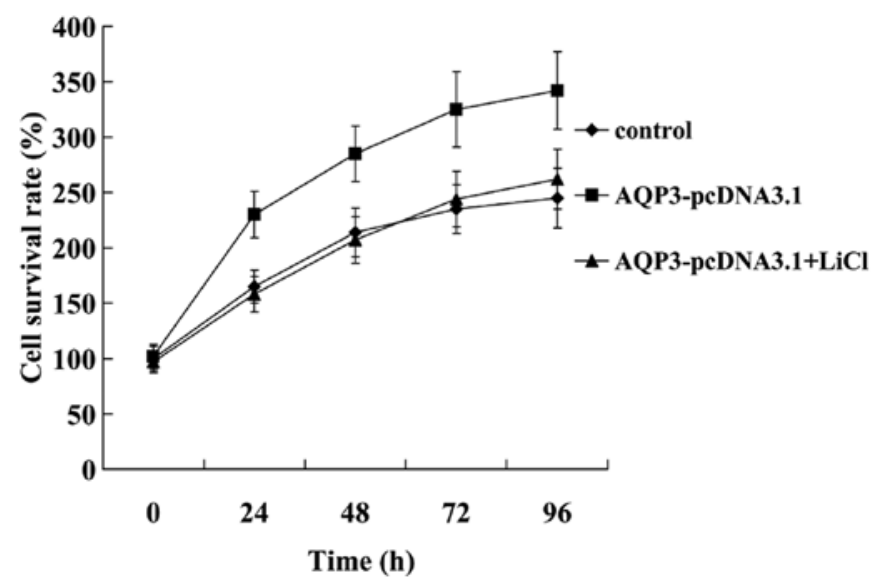

Figure 7. WNT/ $\beta$-catenin signaling activation reverses the effect of aquaporin 3 (AQP3) on human nucleus pulposus cell (hNPC) proliferation. $\mathrm{LiCl}$, lithium chloride.

in the NP tissue samples of patients with lumbar IVD degeneration. This finding is consistent with the report by Ufuk et al which found that AQP-3 immunoreactivity significantly decreased in the NP of aged rats compared with young rats (9).

The abnormal expression of AQP3 has been linked to various disease pathologies $(19,20)$. In the present study, in vitro gainof-function and loss-of-function experiments were performed to verify the effect of AQP3 on IVD degeneration. The IVD consists of three distinct regions: the vertebral endplates (EPs), the AF and the NP (21). NP cells secrete a complex ECM which mainly consists of proteoglycan and fibrillar collagen. The

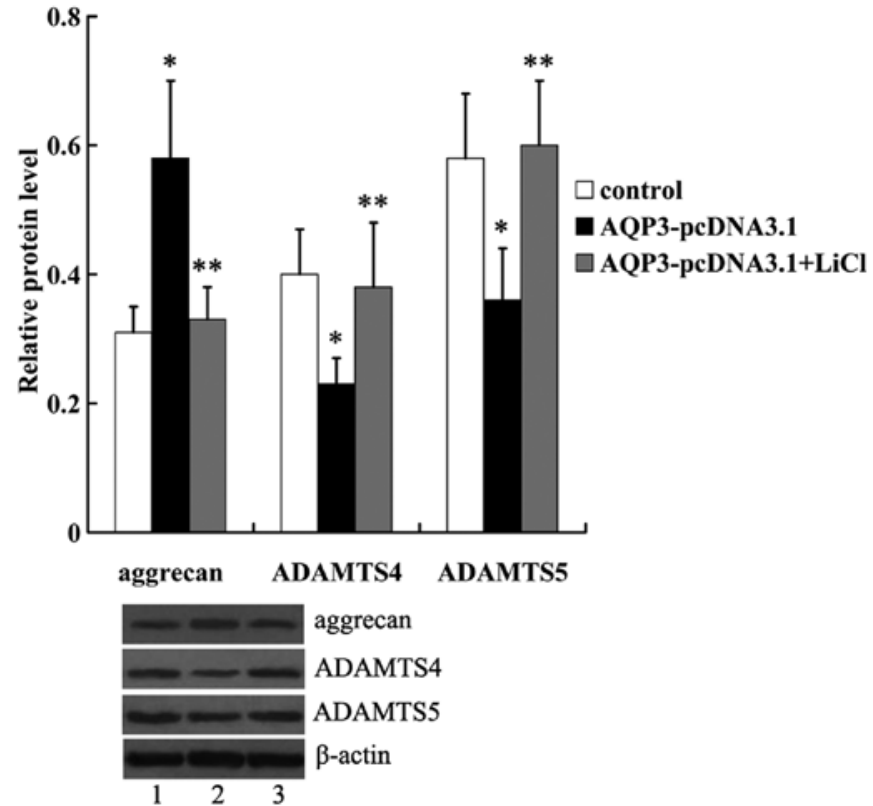

Figure 8 . WNT/ $\beta$-catenin signaling activation reverses the effect of aquaporin 3 (AQP3) on extracellular matrix (ECM) degradation. ${ }^{*} \mathrm{P}<0.05$ compared with the control group; ${ }^{* *} \mathrm{P}<0.05$ compared with the AQP3-pcDNA3.1 group. Lane 1, control group; lane 2, AQP3-pcDNA3.1 group; lane 3, AQP3-pcDNA3.1 + LiCl group. LiCl, lithium chloride; ADAMTS, a disintegrin and metalloproteinase with thrombospondin motifs.

most important elements of disc degeneration are the decline in the number of disc cells and the extensive degradation of the ECM $(22,23)$. Aggrecan is a type of proteoglycan, and it is the major macromolecular component of ECM. The synthesis and degradation of aggrecan are in dynamic equilibrium, maintaining the normal structure and biomechanical function of the IVD (24). ADAMTS4 and ADAMTS5 are the two major aggrecanases, which have the ability to degrade aggrecan (25). It has been demonstrated that the expression of ADAMTSs is increased in the NP of human degenerative disc disease (26).

Our data demonstrated that AQP3 promoted cell proliferation, as determined by an MTT assay. In addition, we found that AQP3 inhibited ECM degradation in hNPCs, as evidenced by the increased expression of aggrecan, and the decreased expression of ADAMTS4 and ADAMTS5. These results indicate that AQP3 exerts protective effects against IVD degeneration.

$\mathrm{Wnt} / \beta$-catenin signaling regulates the proliferation and differentiation of various cell types; therefore, it is involved in tissue degeneration and regeneration $(27,28)$. Several research groups have suggested that $\mathrm{Wnt} / \beta$-catenin signaling contributes to the pathogenesis of IVD degeneration. It has previously been demonstrated that the levels of $\beta$-catenin-positive cells increased as IVD degeneration progressed (29). In a previous study, it was noted that treatment of NP cells with a Wnt/ $\beta$ catenin activator resulted in decreased cell proliferation (15). In addition, activation of $\mathrm{Wnt} / \beta$-catenin signaling induced the expression of matrix metalloproteinases and TGF- $\beta$ in NP cells, leading to an increased breakdown of the matrix, thereby promoting IVD degeneration (16). In the present study, we noted that in hNPCs, Wnt/ $\beta$-catenin signaling was regulated by AQP3. Activation of the $\mathrm{Wnt} / \beta$-catenin signaling pathway attenuated the effect of AQP3 on cell proliferation and ECM degradation in hNPCs. These findings suggest that AQP3 
exerts protective effects against IVD degeneration through the inhibition of Wnt/ $\beta$-catenin signaling.

In conclusion, we have provided in vitro evidence that AQP3 exerts protective effects against IVD degeneration, and these are effected, at least partially, through the inhibition of Wnt/ $\beta$-catenin signaling. This study has improved our understanding of the mechanisms underlying the effects of AQP3 in the progress of IVD degeneration, and suggested a potential strategy for the treatment of IVD degeneration.

\section{References}

1. Podichetty VK: The aging spine: the role of inflammatory mediators in intervertebral disc degeneration. Cell Mol Biol (Noisy-le-grand) 53: 4-18, 2007.

2. Speed C: Low back pain. BMJ 328: 1119-1121, 2004.

3. Takahashi K, Aoki Y and Ohtori S: Resolving discogenic pain. Eur Spine J 17 (Suppl 4): 428-431, 2008.

4. Weber KT, Jacobsen TD, Maidhof R, Virojanapa J, Overby C, Bloom O, Quraishi S, Levine M and Chahine NO: Developments in intervertebral disc disease research: pathophysiology, mechanobiology, and therapeutics. Curr Rev Musculoskelet Med 8: 18-31, 2015.

5. Wang SZ, Rui YF, Lu J and Wang C: Cell and molecular biology of intervertebral disc degeneration: current understanding and implications for potential therapeutic strategies. Cell Prolif 47: 381-390, 2014

6. Isherwood I, Prendergast DJ, Hickey DS and Jenkins JP: Quantitative analysis of intervertebral disc structure. Acta Radiol Suppl 369: 492-495, 1986.

7. Agre P: The aquaporin water channels. Proc Am Thorac Soc 3: $5-13,2006$

8. Zeuthen T and Klaerke DA: Transport of water and glycerol in aquaporin 3 is gated by $\mathrm{H}^{+}$. $\mathrm{J}$ Biol Chem 274: 21631-21636, 1999.

9. Taş U, Caylı S, Inanır A, Ozyurt B, Ocaklı S, Karaca Zİ and Sarsilmaz M: Aquaporin-1 and aquaporin-3 expressions in the intervertebral disc of rats with aging. Balkan Med J 29: 349-353, 2012.

10. Li SB, Yang KS and Zhang YT: Expression of aquaporins 1 and 3 in degenerative tissue of the lumbar intervertebral disc. Genet Mol Res 13: 8225-8233, 2014.

11. Ye S, Wang J, Yang S, Xu W, Xie M, Han K, Zhang B and Wu Z: Specific inhibitory protein Dkk-1 blocking Wnt//-catenin signaling pathway improve protectives effect on the extracellular matrix. J Huazhong Univ Sci Technolog Med Sci 31: 657-662, 2011.

12. Hiyama A, Yokoyama K, Nukaga T, Sakai D and Mochida J: A complex interaction between Wnt signaling and TNF- $\alpha$ in nucleus pulposus cells. Arthritis Res Ther 15: R189, 2013.

13. Kong J, Ma X, Wang T, Ma J, Tian P, Han C, Zang J, Li P and Jiang H: Research progress of Wnt/beta-catenin and nuclear factor-kappa B pathways and their relevance to intervertebral disc degeneration. Zhongguo Xiu Fu Chong Jian Wai Ke Za Zhi 27: 1523-1528, 2013. (In Chinese).

14. Hiyama A, Sakai D, Arai F, Nakajima D, Yokoyama K and Mochida J: Effects of a glycogen synthase kinase-3 $\beta$ inhibitor $(\mathrm{LiCl})$ on c-myc protein in intervertebral disc cells. J Cell Biochem 112: 2974-2986, 2011.
15. Hiyama A, Sakai D, Tanaka M, Arai F, Nakajima D, Abe K and Mochida J: The relationship between the Wnt/ $\beta$-catenin and TGF- 3 /BMP signals in the intervertebral disc cell. J Cell Physiol 226: 1139-1148, 2011.

16. Hiyama A, Sakai D, Risbud MV, Tanaka M, Arai F, Abe K and Mochida J: Enhancement of intervertebral disc cell senescence by $\mathrm{WNT} / \beta$-catenin signaling-induced matrix metalloproteinase expression. Arthritis Rheum 62: 3036-3047, 2010.

17. Mobasheri A, Wray S and Marples D: Distribution of AQP2 and AQP3 water channels in human tissue microarrays. J Mol Histol 36: 1-14, 2005.

18. Hagiwara K, Shinozaki T, Matsuzaki T, Takata K and Takagishi K: Immunolocalization of water channel aquaporins in human knee articular cartilage with intact and early degenerative regions. Med Mol Morphol 46: 104-108, 2013.

19. Meng JH, Ma XC, Li ZM and Wu DC: Aquaporin-1 and aquaporin-3 expressions in the temporo-mandibular joint condylar cartilage after an experimentally induced osteoarthritis. Chin Med J (Engl) 120: 2191-2194, 2007.

20. Shen L, Zhu Z, Huang Y, Shu Y, Sun M, Xu H, Zhang G, Guo R, Wei $\mathrm{W}$ and $\mathrm{Wu} \mathrm{W}$ : Expression profile of multiple aquaporins in human gastric carcinoma and its clinical significance. Biomed Pharmacother 64: 313-318, 2010.

21. Sive JI, Baird P, Jeziorsk M, Watkins A, Hoyland JA and Freemont AJ: Expression of chondrocyte markers by cells of normal and degenerate intervertebral discs. Mol Pathol 55: 91-97, 2002.

22. Roughley PJ, Alini M and Antoniou J: The role of proteoglycans in aging, degeneration and repair of the intervertebral disc. Biochem Soc Trans 30: 869-874, 2002.

23. David G, Ciurea AV, Mitrica M and Mohan A: Impact of changes in extracellular matrix in the lumbar degenerative disc. J Med Life 4: 269-274, 2011.

24. Le Maitre CL,Pockert A, Buttle DJ, Freemont AJ and Hoyland JA: Matrix synthesis and degradation in human intervertebral disc degeneration. Biochem Soc Trans 35: 652-655, 2007.

25. Tian Y, Yuan W, Fujita N, Wang J, Wang H, Shapiro IM and Risbud MV: Inflammatory cytokines associated with degenerative disc disease control aggrecanase-1 (ADAMTS-4) expression in nucleus pulposus cells through MAPK and NF- $\kappa B$. Am J Pathol 182: 2310-2321, 2013.

26. Huang M, Wang HQ, Zhang Q, Yan XD, Hao M and Luo ZJ: Alterations of ADAMTSs and TIMP-3 in human nucleus pulposus cells subjected to compressive load: Implications in the pathogenesis of human intervertebral disc degeneration. J Orthop Res 30: 267-273,2012.

27. Smolders LA, Meij BP, Onis D, Riemers FM, Bergknut N, Wubbolts R, Grinwis GC, Houweling M, Groot Koerkamp MJ, van Leenen $\mathrm{D}$, et al: Gene expression profiling of early intervertebral disc degeneration reveals a down-regulation of canonical Wnt signaling and caveolin-1 expression: implications for development of regenerative strategies. Arthritis Res Ther 15: R23, 2013.

28. Sanges D, Romo N, Simonte G, Di Vicino U, Tahoces AD, Fernández E and Cosma MP: Wnt/ $\beta$-catenin signaling triggers neuron reprogramming and regeneration in the mouse retina. Cell Rep 4: 271-286, 2013.

29. Iwata M, Aikawa T, Hakozaki T, Arai K, Ochi H, Haro H, Tagawa M, Asou Y and Hara Y: Enhancement of Runx2 expression is potentially linked to $\beta$-catenin accumulation in canine intervertebral disc degeneration. J Cell Physiol 230: 180-190, 2015. 